\title{
Functional parcellation of human brain using localized topo- connectivity mapping
}

This paper was downloaded from TechRxiv (https://www.techrxiv.org).

\section{LICENSE}

CC BY 4.0

SUBMISSION DATE / POSTED DATE

31-08-2021 / 01-09-2021

\section{CITATION}

Zhao, Yu; Gao, Yurui; Li, Muwei; Anderson, Adam W.; Ding, Zhaohua; Gore, John C. (2021): Functional parcellation of human brain using localized topo-connectivity mapping. TechRxiv. Preprint. https://doi.org/10.36227/techrxiv.16550097.v1

$\mathrm{DOI}$

10.36227/techrxiv.16550097.v1 


\title{
Functional parcellation of human brain using localized topo-connectivity mapping
}

\author{
Yu Zhao, Yurui Gao, Muwei Li, Adam W. Anderson, Zhaohua Ding, Senior Member, IEEE and John C. \\ Gore
}

\begin{abstract}
The analysis of connectivity between parcellated regions of cortex provides insights into the functional architecture of the brain at a systems level. However, there has been less progress in the derivation of functional structures from voxel-wise analyses at finer scales. We propose a novel method, called localized topoconnectivity mapping with singular-value-decompositioninformed filtering (or filtered LTM), to identify and characterize voxel-wise functional structures in the human brain using resting-state fMRI data. Here we describe its mathematical background and provide a proof-of-concept using simulated data that allow an intuitive interpretation of the results of filtered LTM. The algorithm has also been applied to 7T fMRI data as part of the Human Connectome Project to generate group-average LTM images. Functional structures revealed by this approach agree moderately well with anatomical structures identified by $T_{1}$-weighted images and fractional anisotropy maps derived from diffusion MRI. Moreover, the LTM images also reveal subtle functional variations that are not apparent in the anatomical structures. To assess the performance of LTM images, the subcortical region and occipital white matter were separately parcellated. Statistical tests were performed to demonstrate that the synchronies of fMRI signals in LTMinformed parcellations are significantly larger than those of random parcellations. Overall, the filtered LTM approach can serve as a tool to investigate the functional organization of the brain at the scale of individual voxels as measured in fMRI.
\end{abstract}

Index Terms-Graph signal processing, functional MRI, localized topo-connectivity mapping, functional connectivity, functional parcellation.

\section{INTRODUCTION}

$\mathrm{T}$ O understand the principles and mechanisms of complex brain functions at a systems level, computational models seek to analyze and interpret interactions of components within neurobiological networks [1]. To date, most studies of brain networks have focused on the patterns and dynamics of functional connectivity between parcellated regions of cortex by measuring statistical associations in blood oxygenation level-dependent (BOLD) MRI signals that are related to neural activity [2-4]. The region-level connectivity is commonly represented by a correlation matrix or a topological graph that consists of nodes and edges, which provides an elegant framework for assessments of region-level connectivity profiles, including the 'action-at-a-distance' connections between non-adjacent elements. While numerous studies have demonstrated the effectiveness of the region-level connectivity analysis in network neuroscience [5-7], this approach is unable to capture the spatial organization of individual tissue elements below the level of the selected parcellations. An alternative strategy is to derive functional interactions within 3D brain images using prior knowledge of neural anatomy, assuming brain function and structure are intrinsically related, thus offering the possibility of anatomically constrained, voxel-level analysis of functional connectivity.

Voxel-level functional connectivity has the potential to identify intricate functional structures from groups of individual tissue elements, but in practice it is very challenging due to the limited signal-noise-ratio of fMRI signals and the difficulties of capturing the spatial patterns of functional interactions among the very large number of voxels that could be involved. Recently, principles from the emerging field of graph signal processing (GSP) have been applied in novel voxel-level analyses [8-12]. Under the GSP framework, statistical associations in BOLD signals can be characterized by a topological graph where nodes and linking edges represent image voxels and functional connections between them; mathematical constructs based on graph Laplacians can then be derived, e.g., eigen vectors, or continuous gradients, which depict patterns of spatial continuity of the connectivity in the lattice space [9, 13]. The GSP framework has demonstrated initial successes in delineating functional boundaries within the human brain, which can be further used for parcellations of a functional atlas [10, 14]. However, artificial functional boundaries that often arise from specific geometries of the regions of interest may pose a significant challenge to its applications [9]. Some strategies have been proposed to remedy

Manuscript was submitted on August 18, 2021. This work was supported by National Institutes of Health under Grant R01NS093669 and Grant R01NS113832. (Corresponding author: John C. Gore and Zhaohua Ding).

Yu Zhao and Muwei Li are with Vanderbilt University Institute of Imaging Science and Department of Radiology and Radiological Sciences, Vanderbilt University, TN 37212, United States of America (e-mail: yu.zhao.1@vumc.org and m.li@vumc.org).

Yurui Gao is with Vanderbilt University Institute of Imaging Science and Department of Biomedical Engineering, Vanderbilt University, TN 37212 , United States of America (e-mail: yurui.gao@vanderbilt.edu).

Zhaohua Ding is with Vanderbilt University Institute of Imaging Science, Department of Electrical Engineering and Computer Science and Department of Biomedical Engineering, Vanderbilt University, TN 37212, United States of America (e-mail: zhaohua.ding@vanderbilt.edu).

Adam W. Anderson and John C. Gore are with Vanderbilt University Institute of Imaging Science, Department of Radiology and Radiological Sciences and Department of Biomedical Engineering, Vanderbilt University, Nashville, TN 37212, United States of America (e-mail: adam.anderson@vanderbilt.edu and john.gore@vanderbilt.edu). 
this inherent limitation, but with no proof of their effectiveness in general $[8,9]$. Hence, developing new and more robust methods would be valuable, especially considering that additional information on connectivity at a finer scale is potentially obtainable.

To map functional structures at a voxel level based on topographs, two critical issues may be identified and are addressed in this work. First, signal components shared by separable but adjacent functional structures lead to connections at the boundaries, which could result in blurring of the boundary delineation. Hence, signal filtering should be performed on raw BOLD signals to suppress global signal components and thereby enhance boundary features. A singular value decomposition (SVD) can provide information for such a filtering. Second, capturing local discontinuities in topographic connectivity, or functional boundaries, is key to mapping functional structures, but these may be obscured by non-local connections in topo-graphs. For example, 'action-at-a-distance' functional connections exist between voxels far apart in the anatomical space. This non-localization in the topological graph may confound the local discontinuity of topographic connectivity, potentially again leading to poorly defined functional boundaries. To address this issue, we propose a localization strategy to prune the 'action-at-a-distance' connections. Finally, the functional boundaries that are encoded in the topo-graph are displayed as maps of localized topoconnectivity. The resulting image contrast depicts brain functional structures without the confounding effects from remote connections. We refer to the whole process as localized topo-connectivity mapping with the SVD-informed filtering, and filtered LTM for brevity.

After providing a detailed mathematical formulation of filtered LTM, we describe simulation experiments that illustrate its performance. Then, in vivo studies are reported using resting-state fMRI data acquired by a 7T scanner in the Human Connectome Project (HCP). Functional structures displayed by the resulting LTM images are compared with anatomical structures conveyed by $\mathrm{T}_{1}$-weighted $(\mathrm{T} 1 \mathrm{w})$ images and $\mathrm{FA}$ colored maps. Furthermore, to assess the ability of LTM images to characterize subtle functional structures, we performed two demonstrations of applications. First, LTM images are used to parcellate atlases of subcortical nuclei, which has been proven to be challenging in a recent study [8]. Second, functional structures are parcellated within the occipital white matter. For the two parcellations, rigorous statistical testing of hypotheses is performed to determine whether the synchronies of BOLD signals in LTM-informed parcellations is larger than expected due to chance, where random parcellations are used to estimate a distribution for homogeneity under a null hypothesis.

\section{Methods}

The proposed LTM algorithm consists of two major steps in which filtering of noise and global signal components are followed by an LTM reconstruction (Fig. 1(a)). In the first step, raw time-courses in each volume of interest (VOI) are processed by the singular value decomposition to generate a self-adaptive filter, which is exploited to suppress noise and global signal components in the raw time-courses. In the second step, a contrast metric is derived to capture and visualize spatial continuity patterns of localized topo-connectivity. The contrast mechanism is formulated using differential equations, which are transferred into matrix form to yield analytical solutions, or LTM reconstructions.

\section{A. Filtering of Noise and Global Signal Components.}

The fMRI time-course data from a defined VOI are rearranged into a matrix $(\mathbf{X})$ of dimension $\mathrm{T} \times \mathrm{N}$, where $\mathrm{T}$ denotes the number of time frames and $\mathrm{N}$ the number of voxels within the VOI. SVD of matrix $\mathbf{X}$ is then given by

$$
\mathbf{X}=\mathbf{U} \boldsymbol{\Lambda} \mathbf{P}^{\mathrm{T}},
$$

where $\mathbf{U}(\mathrm{T} \times \mathrm{T})$ and $\mathbf{P}(\mathbf{N} \times \mathbf{N})$ are unitary matrices, and diagonal entries $\left(\sigma_{i}, \sigma_{i}=\boldsymbol{\Lambda}_{i, i}\right)$ of the rectangular diagonal matrix $\boldsymbol{\Lambda}(\mathrm{T} \times \mathrm{N})$ are the singular values of $\mathbf{X}$ in a descending order of magnitude. The columns of $\mathbf{U}\left(\mathbf{U}_{i}\right)$ provide a complete set of orthonormal bases, whereby the time-courses can be represented as a linear combination of the bases, i.e., $\mathbf{X}_{\mathbf{U}}=$ $\mathbf{U}^{\mathrm{T}} \mathbf{X}$. This representation allows an exact reconstruction, that is, the measured signals can be recovered exactly as $\mathbf{X}=\mathbf{U X}_{\mathbf{U}}$. Note that each base vector in $\mathbf{U}$ is associated with a singular value in $\boldsymbol{\Lambda}\left(\mathbf{U}_{i} \rightarrow \sigma_{i}\right)$, and the base vectors in $\mathbf{U}$ are arranged in the same descending order of singular values. An important feature encoded by each base vector in $\mathbf{U}$ is that the base vectors corresponding to larger singular values represent these signal components shared by the voxels in the VOI with larger total weights, compared with the base vectors corresponding to smaller singular values. A typical set of singular values computed from the cortical region of human brain is plotted in descending order of magnitude in Fig. 1(b). Based on SVDinformed component analysis, a self-adaptive filter can be derived to suppress noise and global signal components in raw time-courses. Fig. 1(c) shows three typical time-courses (each $\boldsymbol{x}_{i}$, a column of matrix $\mathbf{X}$ ) projected on to the orthogonal bases $(\mathbf{U})$, where projection coefficients on the first orthogonal bases have significantly larger magnitudes than others. It is well established that neural activities are spatially integrated into multi-regional functional networks. Therefore, signal fluctuations in the time courses evoked by neural events should be shared by multiple locations, or a putative functional network, so in principle these signals project with relatively large coefficients on to the bases associated with larger singular values. In contrast, signal fluctuations caused by noise should have low spatial coherence, and therefore they tend to project on to the bases associated with smaller singular values. To filter the noise in the time courses, we remove the projection coefficients on the bases associated with small singular values, such that the raw signal matrix $\mathbf{X}$ is projected on to a subspace $\left(\mathbf{U}_{\text {trun }}\right)$ that consists of only the bases associated with large 


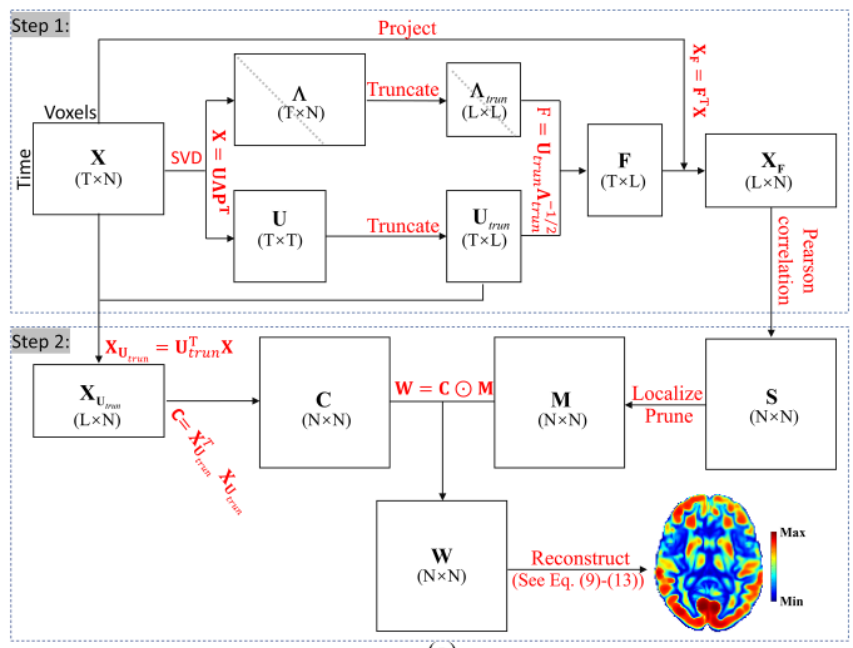

(a)

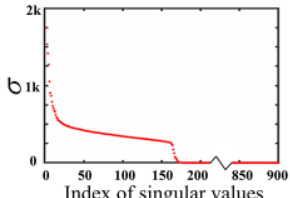

(b)

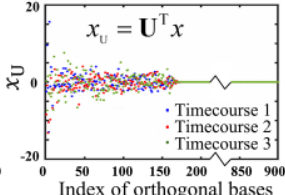

(c)

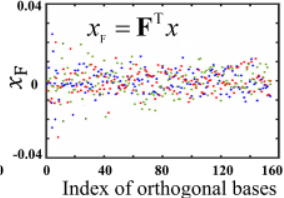

(d)
Fig. 1 Illustration of LTM reconstruction with SVD-informed filtering. (a), detailed algorithm flowchart of LTM reconstruction with SVDinformed filtering. The first major step of the algorithm, SVD-informed filtering, is described as follows. Raw time-courses in a volume of interest (VOI) are first decomposed into singular values and orthogonal bases $\mathbf{U}$ to provide posteriori knowledge for filtering. A filter subspace $\mathbf{F}$ is then constructed based on component analysis of the space $\mathbf{U}$. The raw time-courses are finally projected on to the filter space $F$ to suppress noises and global signal components. The second major step of the algorithm, LTM reconstruction, is described as follows. First, a similarity matrix of F-fingerprint $\mathbf{S}$, defined as pair-wise Pearson correlations among $\mathrm{N}$ voxels in the VOI, is computed (see text). Then, a mask matrix $\mathbf{M}$ is derived from $\mathbf{S}$ with localizing and pruning processes to enhance boundary features in connectivity profile of matrix. Subsequently, $\mathbf{M}$ is applied on the initial connection matrix $\mathbf{C}$ that is calculated from the noise-filtered signals $\mathbf{X}_{\mathbf{U}_{\text {trun }}}$, yielding a boundary-enhanced matrix $\mathbf{W}$. Finally, LTM images are reconstructed with a rationally designed contrast mechanism to capture continuousness patterns of the localized topo-connectivity encoded in the matrix W. (b), singular values plotted in the descending order of magnitude. These values are calculated from fMRI time-courses of the cortical voxels extracted from the dataset of an HCP participant. (c), projection coefficients of three typical time-courses on the orthogonal space $\mathbf{U}$. The projection coefficients on the first orthogonal bases representing global signal components have significantly larger magnitudes than others. The projection coefficients with the indexes from 160 to 900 are very small, which indicate noise components. (d), projection coefficients of the three time-courses on the filter subspace $\mathbf{F}$. Here, the dominant superiority of the projection coefficients on the first orthogonal bases have been reduced. Besides, the noise components have been removed.

singular values. The filtering of noise can be written as

$$
\mathbf{X}_{\mathbf{U}_{\text {trun }}}=\mathbf{U}_{\text {trun }}^{\mathrm{T}} \mathbf{X},
$$

where $\mathbf{U}_{\text {trun }}\left(\mathbf{U}_{\text {trun }}=\left[\mathbf{U}_{1}, \mathbf{U}_{2}, \mathbf{U}_{3}, \ldots, \mathbf{U}_{\mathrm{L}}\right]\right)$ is a truncated form of $\mathbf{U}\left(\mathbf{U}=\left[\mathbf{U}_{1}, \mathbf{U}_{2}, \mathbf{U}_{3}, \ldots, \mathbf{U}_{\mathrm{T}}\right]\right)$, and $\mathrm{L}$ is determined by taking the maximum $\mathrm{k}$ subject to the constraint

$$
\sqrt{\sum_{j \leq \mathrm{k}} \sigma_{j}^{2} / \sum_{i \leq \mathrm{T}} \sigma_{i}^{2}}<1-\xi
$$

where $\xi$ is a selected threshold parameter related to noise level. Subsequently, signal components shared by voxels in the VOI with large total weights are suppressed because these 'global' components would contribute the undesirable back-ground connectivity and weaken the conspicuity of functional boundaries. Thus, after the matrix $\mathbf{X}$ is projected on the subspace $\mathbf{U}_{\text {trun }}$ and multiplied with the matrix

$$
\Lambda_{\text {trun }}^{-1 / 2}=\operatorname{diag}\left(1 / \sqrt{\sigma_{1}}, 1 / \sqrt{\sigma_{2}}, \ldots, 1 / \sqrt{\sigma_{L}}\right),
$$

the resulting projection coefficients associated with large singular values are rescaled to relatively small values. As shown in Fig. 1(d), the dominance of the projection coefficients on the first orthogonal bases have been reduced. Filtering of the noise and the global signal components simultaneously can be reformulated by

$$
\mathbf{X}_{\mathbf{F}}=\mathbf{F}^{\mathrm{T}} \mathbf{X}
$$

where $\mathbf{F}=\mathbf{U}_{\text {trun }} \boldsymbol{\Lambda}_{\text {trun }}^{-1 / 2}$. We refer to $\mathbf{X}_{\mathbf{F}}$ as an F-fingerprint matrix. The columns of $\mathbf{F}\left(\mathbf{F}_{i}\right)$ are a set of orthogonal bases, subject to $\mathbf{F}_{i}^{\mathrm{T}} \mathbf{F}_{j}=0,(i \neq j)$ and $\left\|\mathbf{F}_{i}\right\|_{2}=1 / \sqrt{\sigma_{i}}$. Note that the variance of projection coefficients of $\mathbf{X}$ on the $i$-th base of $\mathbf{U}$ $\left(\mathbf{U}_{i}\right)$ is $\sigma_{i}$, and the variance becomes $\sqrt{\sigma_{i}}$ on the $i$-th base of $\mathbf{F}$ $\left(\mathbf{F}_{i}\right)$ and thus the sorting order of the variances is maintained. By this operation, the dominance of the projection coefficients on the first orthogonal bases are reduced, but the sorting order of variances is preserved so that the bases of $\mathbf{F}$ associated with large singular values still carry large weights in characterizing connectivity profiles. This avoids the potential that signal components that account for only a small portion of total variance in the connectivity characterization could become dominant.

\section{B. LTM Reconstruction}

To reveal the functional connectivity between voxels in 3D space using the GSP framework, a voxel-level topological graph is derived based on the use of the F-fingerprints, or the filtered time-courses. We denote the graph as $\mathbf{G}=\{\mathbf{V}, \mathbf{E}, \mathbf{W}\}$, which consists of a finite set of vertices $\mathbf{V}$ with $|\mathbf{V}|=\mathrm{N}$, a set of edges $\mathbf{E}$, and a weighted connection matrix $\mathbf{W}$. The entry $\mathbf{W}_{i, j}$ represents the connection strength of edge $\boldsymbol{e}=(i, j)$ that connects vertices $i$ and $j$. On the vertices of the graph, a vector $\mathbf{f} \in \mathbb{R}^{N}$ is defined to represent the intensity of the LTM image, where the $i$-th component of the vector, $f(i)$, represents the value at the $i$-th vertex in $\mathbf{V}$. Note that the fMRI time-courses on vertices are measured data while the $1 \mathrm{D}$ vector $\mathbf{f}$ is a set of auxiliary quantities to be derived computationally (see details below), which is finally represented in $3 \mathrm{D}$ space to characterize spatial continuity patterns of localized topo-connectivity. An initial connection matrix $\mathbf{C}$ of a graph is computed based on the noise-filtered signals $\left(\mathbf{X}_{\mathbf{U}_{\text {trun }}}\right.$ ) associated with pairs of voxels in the VOI, which is derived as

$$
\mathbf{C}=\mathbf{X}_{\mathbf{U}_{\text {trun }}}^{\mathrm{T}} \mathbf{X}_{\mathbf{U}_{\text {trun }}}
$$


To enhance boundary features encoded in the denoised connectivity matrix $\mathbf{C}$, sparsification is performed on $\mathbf{C}$ to yield the final weighted connection matrix $\mathbf{W}$ as follows. First, similarity between each voxel pair is calculated by Pearson correlation of their F-fingerprints such that pair-wise similarities among $\mathrm{N}$ voxels can be represented by a symmetric matrix is $\mathbf{S} \in \mathbb{R}^{N \times N}$. Note that a group-consensus similarity matrix can be obtained by averaging over individual subjects aligned in a common space to achieve topo-connectivity mapping at the group level. A mask matrix $\mathbf{M}$ for the enhancement of boundary features can then be derived from $\mathrm{S}$ by applying two constraints: (1) the connections of the vertex $i$ are localized in its neighborhood $\mathcal{N}_{i}$ defined by the cubic lattices of size $5 \times 5 \times 5$ centered at the vertex $i$; (2) the weights of the localized connections with a Euclidean distance greater than $\varepsilon$ are set to zero, where $\varepsilon$ is defined as the minimum value required for the graph to stay connected [15]. Formally, the localizing and pruning process can be formulated as

$$
\mathbf{M}_{i, j}=\left\{\begin{array}{cc}
1 & \text { if }\left\|\mathbf{S}_{i .}-\mathbf{S}_{j .}\right\|<\varepsilon \text { and } j \in \mathcal{N}_{i}, \\
0 & \text { otherwise }
\end{array}\right.
$$

where $\mathbf{S}_{i \text {. and }} \mathbf{S}_{j .}$ are the $i$-th column and the $j$-th column of $\mathbf{S}$ that represent the vectors of similarity between vertex $i$ and $j$ and all the vertices in $\mathbf{V}$, respectively; and $\left\|\boldsymbol{S}_{i .}-\mathbf{S}_{j}.\right\|$ denotes the Euclidean distance between the two vectors. Finally, this mask matrix is applied on the connection matrix $\mathbf{C}$ to yield the weighted connection matrix $\mathbf{W}$ to be used for the LTM reconstruction, which is derived as

$$
\mathbf{W}=\mathbf{C} \odot \mathbf{M} .
$$

At the beginning of LTM reconstruction, the auxiliary quantities $\mathbf{f}$ are initialized to the state $f_{0}(i)=c(i=$ $1,2, \ldots, N)$, hence initial values of LTM images are the same at all positions. Then, $\mathbf{f}$ evolves with time to yield LTM images, in which image values are modulated by a rationally designed mechanism such that the resulting image contrasts convey the spatial continuity patterns of the localized topo-connectivity that is encoded in the matrix $\mathbf{W}$. The rationally designed mechanism of the time-dependent evolution includes two principles: a spatially uniform decay and locally connectivityspecific propagation. The former takes account that BOLD signals decay at a constant decay rate $r$ for all vertices. The latter requires that the value on the vertex $i$ diffuses away at a localized-connectivity-dependent rate. Note that the voxels belonging to a connectivity community would tend to have similar values as propagations of intensities of the voxels are coupled with each other. With these two principles, the timedependent evolution of the signal on a single vertex $i$ can be described by a differential equation,

$$
\frac{d f(i, t)}{d t}=-r \cdot f(i, t)+\sum_{j \in \mathcal{N}_{i}} \mathbf{W}_{i, j} f(j, t) .
$$

The time-dependent evolution of the signals on all the vertices of the graph can be formulated in a compact form as

$$
\frac{d \mathbf{f}(t)}{d t}=\mathbf{J} \cdot \mathbf{f}(t)
$$

where $\mathbf{J}=\mathbf{W}-r \cdot \mathbf{I}$, and $\mathbf{I}$ is an identity matrix of $\mathbf{N} \times \mathbf{N}$.

The graph operator $\mathbf{J}$ is a real symmetric matrix, thus it has a complete set of orthonormal eigenvectors $\left\{\boldsymbol{u}_{\ell}\right\}_{\ell=1,2, \ldots, N}$ and associated eigenvalues $\left\{\lambda_{\ell}\right\}_{\ell=1,2, \ldots, N}$, satisfying $\mathbf{J} \boldsymbol{u}_{\ell}=\lambda_{\ell} \boldsymbol{u}_{\ell}$. Based on these eigenvectors, the graph signal $\mathbf{f}$ can be transformed into a spectral representation,

$$
\hat{\mathbf{f}}(\ell)=\sum_{i=1}^{N} \boldsymbol{u}_{\ell}^{\mathrm{T}}(i) \mathbf{f}(i)=\boldsymbol{u}_{\ell}^{\mathrm{T}} \mathbf{f},(\ell=1,2, \ldots, N)
$$

This spectral representation allows an exact reconstruction, i.e., the signal $\mathbf{f}$ can be recovered as

$$
\mathbf{f}=\sum_{\ell=1}^{N} \hat{\mathbf{f}}(\ell) \boldsymbol{u}_{\ell}
$$

Accordingly, the analytic solution of Eq. (10) with an initial state signal $\mathbf{f}_{0}(\mathrm{t}=0)$ can be derived as

$$
\tilde{\mathbf{f}}(t)=\sum_{\ell=1}^{N} e^{\lambda_{\ell} t} \hat{\mathbf{f}}_{0}(\ell) \boldsymbol{u}_{\ell}=\sum_{\ell=1}^{N} e^{\lambda_{\ell} t} \boldsymbol{u}_{\ell}^{\mathrm{T}} \mathbf{f}_{0} \boldsymbol{u}_{\ell} .
$$

Given an appropriate value of parameter $r$ and the initial state $f_{0}(i)=c(i=1,2, \ldots N)$, the signal $\tilde{\mathbf{f}}(t)$ at time point $\mathrm{t}$ conveys the image value for characterizing the spatial continuity pattern of the localized topo-connectivity. Based on analysis of Eq. (9), it can be inferred that the image intensity of a vertex depends on the total connecting weight of this vertex. As a result, the values of isolated vertices and those between connected vertex communities (e.g., functional networks) would have lower intensities than those within a connected vertex community. Hence, this feature would lead to contrast valleys along the underlying boundaries between two connectivity communities, which naturally allows the boundaries in reconstructed images to be visualized. In addition, the propagation of signal at a vertex is modulated by the total connecting weights with respect to its $5 \times 5 \times 5$ neighbors as well as signal intensities of these neighbors, such that the propagations of signals are spatially coupled and thus the vertices within a community tend to have similar values. Furthermore, vertices across communities tend to have different values because different vertex communities tend to have specific connectivity levels. Therefore, the image values at the community boundaries will lead to rapid contrast changes across the communities, which offers an additional mechanism to distinguish different communities. Taken together, the two mechanisms are involved in delineations of functional boundaries, namely 'valleys' and 'cliffs', where 'valleys' represents low contrasts on the connectivity boundaries, and 'cliffs' represents rapid contrast changes across the connectivity 
communities. In this study, the proposed LTM reconstruction is conducted after SVD-informed filtering, and thus is referred to as filtered LTM reconstruction.

\section{Proof of Concept with Simulated Data}

Simulated 4D data were generated to demonstrate the concept of characterizing spatial patterns of localized topoconnectivity using the proposed filtered LTM reconstruction. The data were simulated with spatial patterns of topoconnectivity specified as follows. Briefly, time-courses with 900 time frames were assigned to a 3D phantom of dimensions $81 \times 81 \times 5$, along the first two of which artificial spatial patterns were synthesized. The synthesized 2D spatial patterns were defined by constructing ten areas such that time-courses within each area were identical and across areas were different. Subsequently, uncorrelated noise time-courses were added to the time-courses of the synthetic patterns to achieve a signal-tonoise ratio (SNR) of 0.05 . The correlational relationships of the time-courses within each area and between the areas were then computed and represented by a correlation matrix. Finally, the proposed filtered LTM reconstruction was implemented on the simulated data, to validate its ability to recover the spatial patterns of synthesized topo-connectivity.

\section{Filtered LTM Reconstruction of Human fMRI Data}

\section{1) Data and preprocessing}

Human fMRI data were sourced from the Human Connectome Project (HCP) database and included part of the HCP S1200 cohort which involved healthy young adults ranging between 22 and 37 years old. HCP datasets used in this study included 153 participants collected on a Siemens Magnetom 7T MR scanner that passed quality control criteria. HCP datasets used in this study include three imaging modalities: Resting-state fMRI, $\mathrm{T}_{1} \mathrm{~W}$ MRI and Diffusion MRI. Parameters of the three MRI scans are described in [16]. Minimally preprocessed volumetric fMRI data were downloaded from the HCP repository. The minimal preprocessing pipeline for the fMRI modality includes removing spatial distortions, realigning volumes to compensate for subject motion, registering the fMRI data to the structural $\mathrm{T} 1 \mathrm{w}$ images, reducing the bias field, normalizing the $4 \mathrm{D}$ image to a global mean, and masking the data with a final brain mask. Further preprocessing before the filtered LTM reconstruction included three steps as follows. First, spatial smoothing was performed with a Gaussian smoothing kernel of 4-mm full width at half maximum. Second, time-courses were bandpass filtered to include frequencies spanning from 0.01 to $0.1 \mathrm{~Hz}$. Finally, the means of the filtered time-courses were subtracted, and the data then normalized to unit variance. The fMRI dataset was separated into two groups: the first 140 subjects for a primary dataset of LTM reconstruction and the remaining 13 subjects for an independent validation dataset.

\section{2) LTM reconstruction of functional volumes}

The filtered LTM reconstruction on a group level was conducted on selected brain cortex and a composite region including white and subcortical gray matters separately, which is described in Section I in Supplementary material. The four key parameters of the LTM reconstructions $\{\xi, r, c, t\}$ were determined according to Section II in Supplementary material. The reconstructed LTM images convey group-average functional structures, which were interpreted by reference to group-consensus $\mathrm{T}_{1} \mathrm{w}$ images and DTI fractional anisotropy (FA) colored images from a representative subject. The groupconsensus $T_{1} \mathrm{~W}$ images were obtained by averaging $140 \mathrm{~T}_{1} \mathrm{~W}$ images from subjects in the primary dataset after a registration to the MNI152 space. FA colored images used as a reference were collected from only one subject since accurate registration is challenging due to distortions stemming from eddy currents and inhomogeneities of the main magnetic field in the DTI acquisition $[17,18]$.

\section{3) Parcellation of nuclei and occipital white matter}

Based on the LTM-derived functional structures, parcellation was performed for subcortical nuclei and occipital white matter separately. A key marker of parcellation validity is the withinparcel synchrony of fMRI time-courses, which was defined as the variance explained by the first principal component estimated using PCA. For each parcellation, the synchrony was calculated for each hemisphere then summed. To determine whether the synchrony of fMRI time-courses in LTM-informed parcellation was larger than expected due to chance, 200 random parcellations were created to estimate a distribution of synchronies for the null hypothesis of statistical testing. A schematic of the related inferential statistics is given in Fig. 2. The random parcellations were generated by the following steps: (1) dilation was performed on the LTM-informed parcellation by three layers of voxels to generate a confining mask; (2) a seed voxel was placed randomly in the LTMinformed parcellation; (3) voxels grew randomly from the seed voxel in the confining mask, until the total number of voxels matched that of the LTM-informed parcellation. Specifically, new voxels iteratively grew at immediate neighbors of existing voxels with equal probabilities. In each iteration, only one new voxel was grown; the voxel was removed if outside the confining mask and any holes within the grown volume were filled using simple morphological operations. To ensure spatial correspondences between random and LTM-informed parcellations, the random parcellation was discarded if it overlapped with less than half of its corresponding LTMinformed parcellation. Finally, a statistical testing with the null hypothesis, i.e., the distribution of synchronies estimated by the 200 random parcellations, was performed. Briefly, for each parcellation, the overall synchrony of fMRI signals was first calculated by summing up the synchronies of the independent validation dataset of 13 subjects. The synchronies were then normalized with respect to the mean synchrony across the set of random parcellations. The fMRI time-courses in LTMinformed parcellation (alternative hypothesis) were deemed synchronous if the null hypothesis was rejected $(\mathrm{P}<0.05)$. 


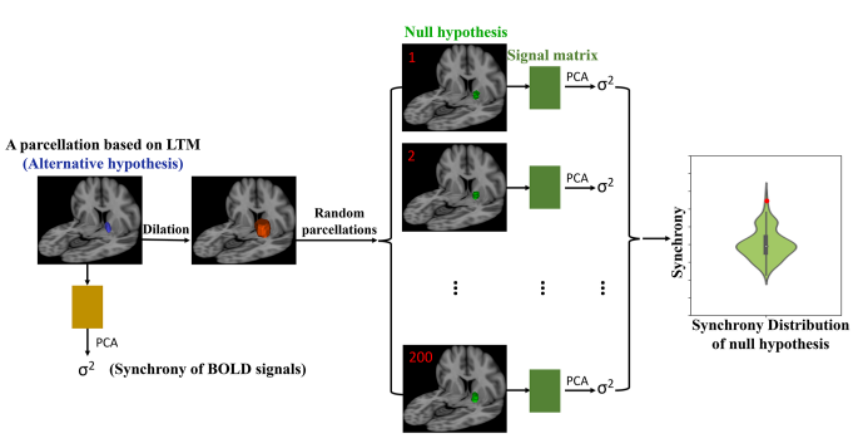

Fig. 2. Schematic of synchrony estimation. A key marker of parcellation validity is within-parcel synchrony of functional signals, which is defined as the variance explained by the first principal component of this matrix that was estimated using PCA. To determine whether the synchrony of LTM-informed parcellations was larger than expected due to chance, 200 random parcellations are created to estimate a distribution for synchrony for the null hypothesis. A violin plot is used to display inferential statistics for the parcellation. In the violin plot, red dots indicate synchrony value of LTM informed parcellation. Bottom and top edges of the narrow box in violin plot indicate 25th and 75th percentiles of the distribution, respectively. The central mark indicates the median. The whiskers extend to the most extreme data points that are not considered outliers $(1.5 \times$ interquartile range $)$.

\section{Results}

\section{A. Experiments with Simulated Data}

Proof-of-concept results with simulated data are demonstrated in Fig. 3, which shows the patterns of the simulated areas, the effects of SVD-informed filtering, and the reconstructed LTM images. In Fig. 3(a), the spatial patterns of the simulated phantom were defined by ten non-overlapping areas (denoted alphanumerically and in different colors), each of which was constructed with synthetic time-courses that encoded connectivity patterns. The time-courses across the ten color-encoded areas were correlated at various levels, which are displayed in Fig. 3(b) by a connectivity matrix representing Pearson correlations between the raw time-courses. Based on this correlation or connectivity matrix, the ten areas were separated into three groups, namely, $\{\mathrm{A} 1, \mathrm{~A} 2, \mathrm{~A} 3\},\{\mathrm{B} 1, \mathrm{~B} 2$, $\mathrm{B} 3\}$ and $\{\mathrm{C} 1, \mathrm{C} 2, \mathrm{C} 3, \mathrm{D}\}$, with specific properties including: each area within $\{\mathrm{A} 1, \mathrm{~A} 2, \mathrm{~A} 3\}$ had a different intra-area connectivity profile; each area in $\{\mathrm{A} 1, \mathrm{~A} 2, \mathrm{~A} 3\}$ and $\{\mathrm{B} 1, \mathrm{~B} 2$, B3\} had negligible inter-area connectivity with all its adjacent areas; the time-courses bore common global components in $\{\mathrm{C} 1, \mathrm{C} 2, \mathrm{C} 3, \mathrm{D}\}$, and thus these areas had relatively large connectivity with each other, which is shown in the $4 \times 4$ block of higher connectivity in the lower right corner in Fig. 3(b).

The final LTM images reconstructed without and with SVDinformed filtering are shown in Fig. 3(e)\&3(f), respectively. From both reconstructions, the ten areas with pre-defined patterns could be identified using the 'valleys or cliffs' for boundary delineation. Both reconstructed images show that contrasts are proportional to the connectivity level, which is particularly pronounced in the area group $\{\mathrm{A} 1, \mathrm{~A} 2, \mathrm{~A} 3\}$. The effects of SVD-informed filtering are demonstrated in Fig. 3(c), which shows the similarity matrix computed from the pairwise correlations of filtered time-courses, or F-fingerprints. It can be observed that, after SVD-informed filtering, the inter-area connectivity between the areas in $\{\mathrm{C} 1, \mathrm{C} 2, \mathrm{C} 3, \mathrm{D}\}$ have been

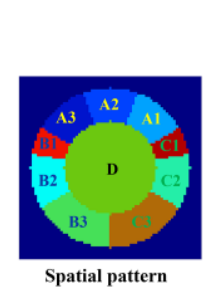

(a)

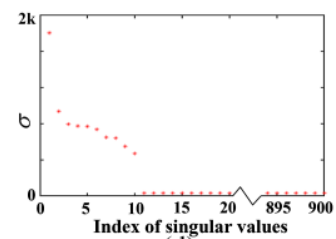

(d) (b)

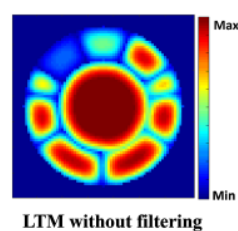

(e)

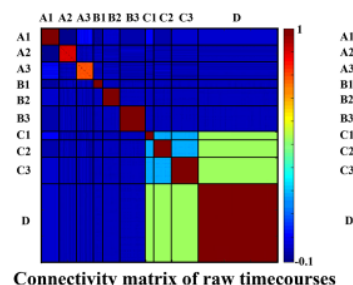

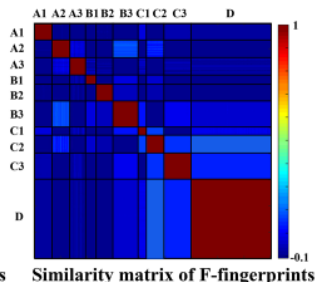

(c)

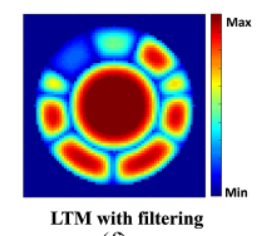

(f)
Fig. 3. Experiments with simulated data. (a), spatial patterns of a simulated phantom, which contains ten distinct areas marked in different colors. (b), connectivity matrix derived from time-courses in the areas of (a). The connectivity matrix consists of pair-wise Pearson correlations between the raw time-courses. Based on this matrix, the ten patterndefined areas are separated into three groups, i.e., $\{A 1, A 2, A 3\},\{B 1$, B2, B3 $\}$ and $\{C 1, C 2, C 3, D\}$. (c), similarity matrix (S) derived from Ffingerprints. This matrix demonstrates that the SVD-informed filtering enhances the connectivity between the voxels within each area of the group $\{A 1, A 2, A 3\}$, and reduces the connectivity between the voxels across areas of the group $\{C 1, C 2, C 3, D\}$. (d), singular values plotted in the descending order of magnitude. Note that the relatively large singular values are used for the SVD-informed filtering. (e) and (f), reconstructed LTM images and the effects of SVD-informed filtering. In the LTM image reconstructed without the filtering $(e)$, the contrasts of the areas of $\{A 1, A 2, A 3\}$ are positively related with the connectivity levels, and the presence of common global components in the areas of $\{C 1, C 2, C 3, D\}$ causes blurring effects around the boundaries. The blurring effects in the areas of $\{C 1, C 2, C 3, D\}$ are absent in the LTM image reconstructed with the filtering $(\mathrm{f})$.

reduced to relatively low levels. The threshold parameter $\xi$ for the filtering was determined by taking relatively large singular values, i.e., the first ten singular values int this case, as seen in Fig. 3(d). In the LTM image reconstructed without filtering (Fig. 3(d)), there are obvious blurring effects on boundaries of the areas in the group $\{\mathrm{C} 1, \mathrm{C} 2, \mathrm{C} 3, \mathrm{D}\}$, which is presumably induced by the common global components these areas share. In Fig. 3(f), the blurring effects on the boundaries of the same areas are eliminated by the filtering. An additional nonnegligible phenomenon present in both reconstructed LTM images is that the average LTM contrast of each area in $\{\mathrm{C} 1$, $\mathrm{C} 2, \mathrm{C} 3, \mathrm{D}\}$ and $\{\mathrm{B} 1, \mathrm{~B} 2, \mathrm{~B} 3\}$ is positively correlated with the number of voxels it contains. This phenomenon arises because the signal propagations of voxels belonging to a connectivity community are coupled in LTM reconstruction. Specifically, the voxels on boundaries with poor connections could reduce image intensities in the whole connectivity community, which is dependent on the proportion of voxels on boundaries in the connectivity community.

\section{B. Experiments with in vivo data}

Fig. 4 shows group-average LTM images reconstructed with the fMRI dataset collected from 140 participants in HCP, as well as group-average $\mathrm{T}_{1} \mathrm{~W}$ images and FA colored maps from a representative participant. In the LTM images, the patterns of 'valleys or cliffs' show functional boundaries, so that 


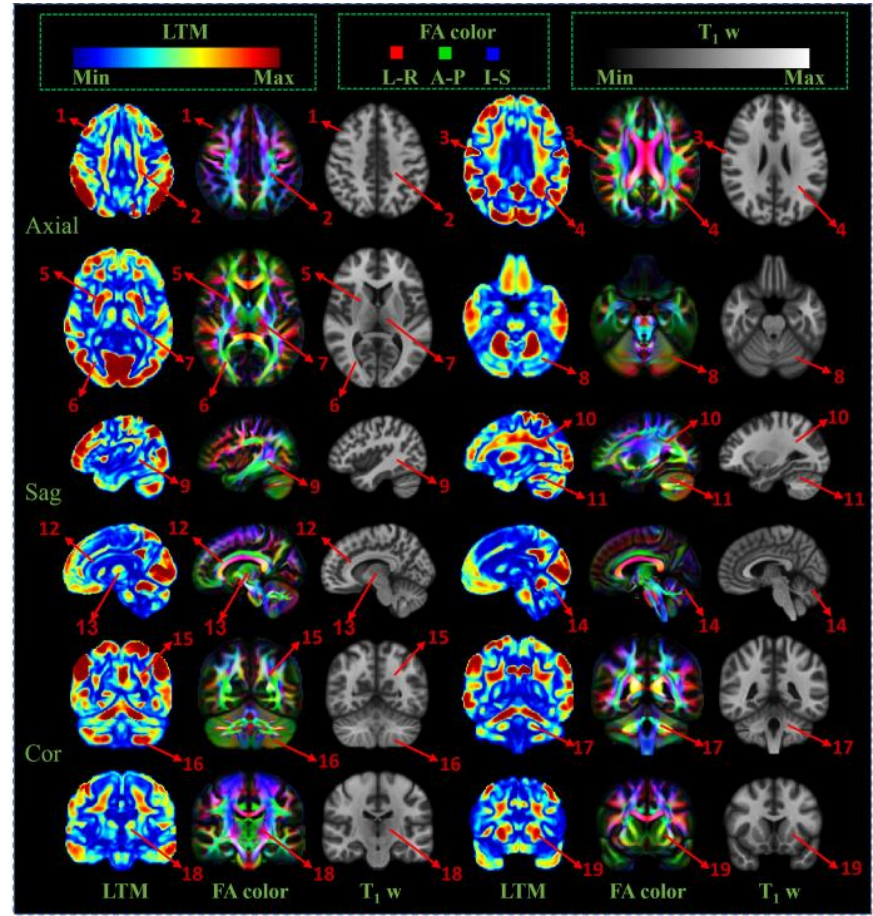

Fig. 4. Group-average LTM images reconstructed from 140 participants, group-average T1w images obtained by averaging over the 140 participants and FA maps from one representative participant. Functional structures revealed by LTM reconstruction are compared with anatomical structures conveyed by $\mathrm{T}_{1}$ relaxation and diffusion anisotropy. In the LTM images, functional boundaries can be identified in the LTM images using the 'valleys or cliffs' mechanisms. Furthermore, based on delineation of the functional boundaries, connected vertices can be parcellated to yield connectivity communities, or functional areas. Some typical connectivity communities are indicated by red arrows and concomitant Arabic numerals (1-19), which are analyzed in the main text.

connectivity communities can be further identified based on the boundary delineations. In Fig. 4, several selected connectivity communities are indicated by red arrows and numerical labels. For the cerebral cortex, these connectivity communities correspond with anatomical structures in grey matter as apparent in the $T_{1} \mathrm{w}$ images. Two connectivity communities indicated by the labels 1 and 3 align with white matter and agree moderately with anatomical structures within the FA colored map. It is noticeable that only some regions of white matter have high intensities in LTM images. Some typical connectivity communities of white matter are indicated by the labels $\{2,4$, $6,9,10,12,15\}$. In the cerebral subcortex, as indicated by the labels $\{5,7,13,18,19\}$, connectivity structures in LTM images clearly correspond with structures that can be identified in $\mathrm{T}_{1} \mathrm{~W}$ images, but subtle structures within the LTM images (e.g., subdivisions of the thalamus indicted by $\{7\}$ ) are not seen in the $\mathrm{T}_{1}$ or FA colored maps. In the cerebellum, LTM images show connectivity communities labelled as $\{8,11,14,16,17\}$. Overall, functional structures derived from LTM images show a moderate agreement with anatomical structures depicted in the $\mathrm{T}_{1} \mathrm{~W}$ images and the FA colored maps. For additional visualization, we display LTM-based connectivity communities on two types of brain surfaces in Fig. 5 (5(a) corresponding to ICBM152 brain surface, and 5(b) corresponding to ICBM152 (a)

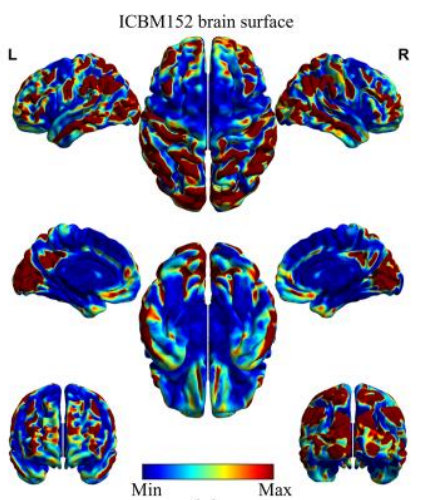

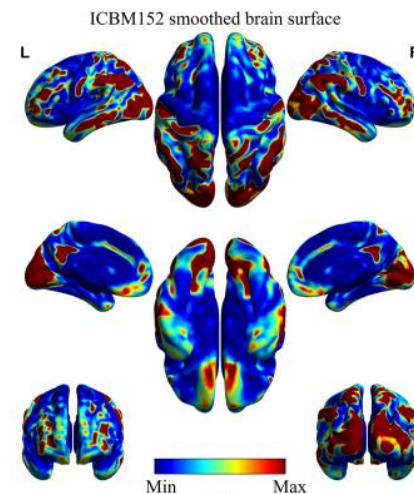

(b)
Fig. 5. Connectivity patterns of LTM reconstruction on ICBM152 brain surface (a), and ICBM152 smoothed brain surface (b). Clusters of voxels in red colors represent functional areas, wherein associated BOLD signals are relatively more synchronous than those in blue colors.

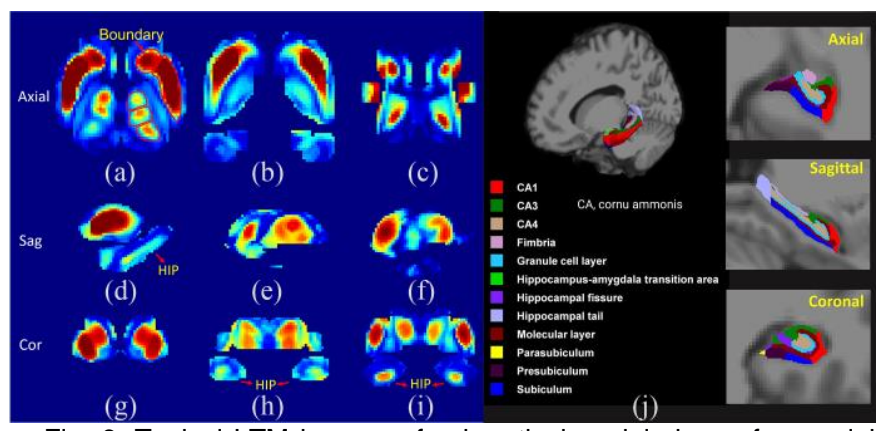

Fig. 6. Typical LTM images of subcortical nuclei shown from axial, coronal and sagittal perspectives (a-i) and anatomical structures of hippocampus (j). To display detailed structures, the LTM images of subcortical nucleus are segmented from the LTM images of whole brain with a coarse subcortex mask. Using the 'valleys or cliffs' mechanisms, functional boundaries can be delineated in the LTM images. Some typical boundaries are indicated by red lines in a. Note that the centrically stratified structures of hippocampus (indicated in $\mathrm{d}, \mathrm{h}$ and i) agree well with anatomical structures of hippocampus (j) that is reproduced from [19]; use permitted under the Creative Commons Attribution License CC BY 4.0.

smoothed brain surface). Clusters of voxels in red colors represent functional areas on the surfaces, in which associated BOLD signals are relatively more synchronous that those in blue colors.

\section{1) Parcellation of subcortical nuclei}

LTM images of subcortical nuclei are shown in axial, coronal and sagittal sections in Fig. 6(a)-6(i), which are segmented from the whole brain with a course, subcortical mask. Based on the apparent "valleys and cliffs", functional boundaries can be delineated in the LTM images (e.g., as indicated by red lines in Fig. 6(a)). Voxels with high intensities can be clustered into connectivity communities, whereas voxels with low intensities have BOLD signals that are relatively asynchronous. As indicated by red arrows in Fig. 6(d), 6(h) and 6(i), numerous voxels around the core of the hippocampus have low intensities, which suggests a centrically stratified structure that has been reported in a previous study [19] and is redisplayed in Fig. 6(j). Using the boundary delineations of 3D LTM images, parcellation of the subcortical region can be used to generate an atlas, as shown in Fig. 7(a). This atlas identifies well-known 


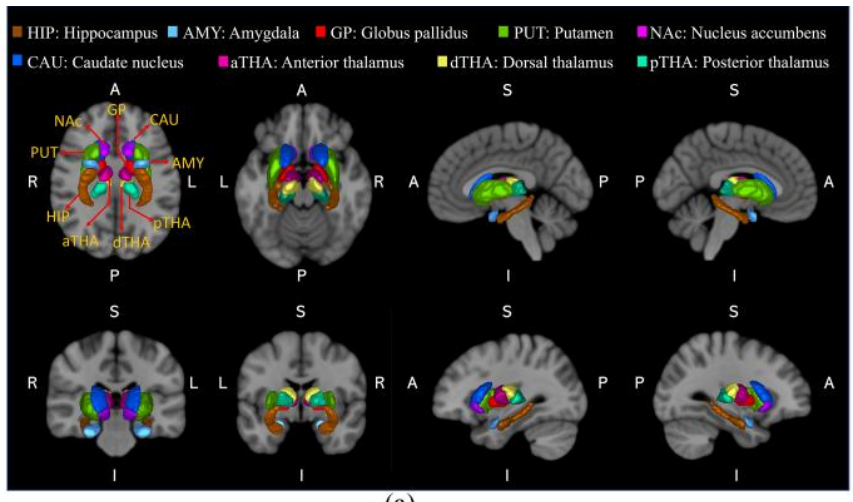

(a)

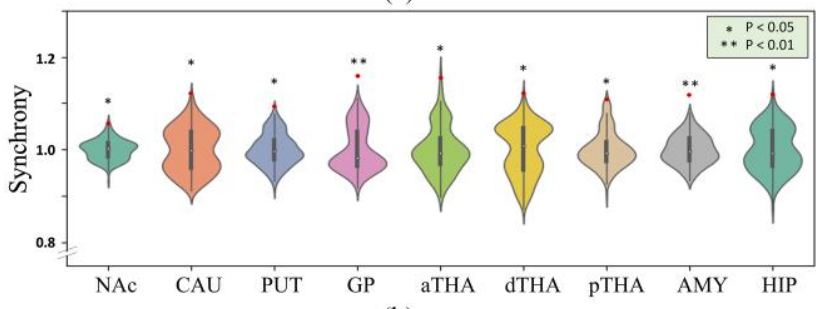

(b)

Fig. 7. Parcellation atlas of subcortical nuclei and synchrony assessment. (a), parcellation atlas of subcortical nuclei. To show the detailed structures, the atlas is displayed from multiple perspectives, which are indicated by the direction labels (L: left, R: right, A: anterior, $P$ : posterior, I: inferior, S: superior). This atlas recapitulated well-known anatomic nuclei, namely, the nucleus accumbens, the globus pallidus, the caudate and the putamen, the amygdala, the hippocampus, the thalamus. Note that the thalamus is subdivided into the anterior thalamus, the dorsal thalamus and the posterior thalamus. (b), results of synchrony assessment. In violin plot, red dots indicate synchrony values of LTM informed parcellation. Bottom and top edges of the narrow box in violin plot indicate 25th and 75th percentiles of the distribution, respectively. The central mark indicates the median. The whiskers extend to the most extreme data points that are not considered outliers (1.5 $\times$ interquartile range). Statistical analysis demonstrates that BOLD signals in all LTM-informed parcellations are significantly more synchronous than expected due to chance $(P<0.05$ : the nucleus accumbens, the caudate nucleus, the putamen, the anterior thalamus, the dorsal thalamus, the posterior thalamus and the hippocampus; $\mathrm{P}<0.01$ : the globus pallidus and the amygdala)

anatomic nuclei, namely, the nucleus accumbens, the globus pallidus, the caudate nucleus, the putamen, the amygdala, the hippocampus, and the thalamus. Note that the thalamus is subdivided into the anterior thalamus, the dorsal thalamus and the posterior thalamus. Validation of these parcellations was conducted on a separate dataset of 13 subjects. As shown in Fig. 7(b), statistical analysis demonstrates that fMRI time-courses in all LTM-informed parcellations of the nuclei are significantly more synchronous than expected due to chance $(\mathrm{P}<0.05$ : the nucleus accumbens, the caudate nucleus, the putamen, the anterior thalamus, the dorsal thalamus, the posterior thalamus and the hippocampus; $\mathrm{P}<0.01$ : the globus pallidus and the amygdala).

\section{2) Parcellation of occipital white matter}

Fig. 8 shows a functional parcellation of occipital white matter. Three typical LTM images are displayed in axial, coronal and sagittal sections in Fig. 8(a)-8(c), respectively, wherein clear boundaries can be observed between the gray matter and the white matter. With delineation of the boundaries, occipital white matter was then parcellated on the LTM images.

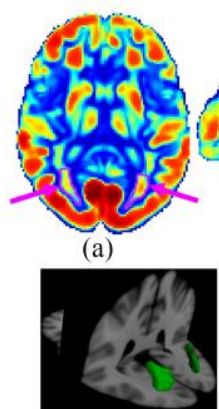

(d)

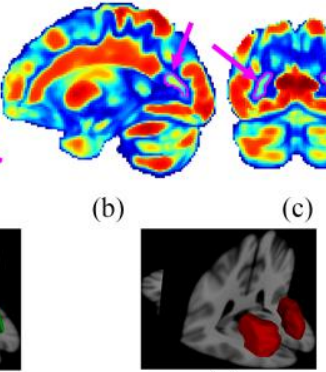

(e)

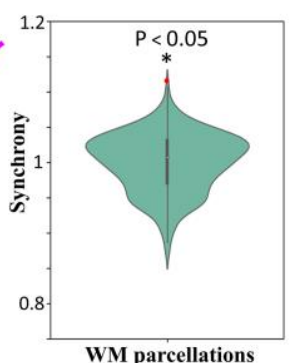

(f)
Fig. 8. Parcellation of occipital white matter and synchrony assessment. (a-c), three typical LTM images displayed from axial, coronal and sagittal perspectives, respectively. In the LTM images, clear boundaries can be distinguished between the gray matter and the white matter. (d\&e), parcellation of occipital white matter and a confine mask for generating the random parcellations of null hypothesis. (f), synchrony assessment of the LTM-informed parcellation of occipital white matter. In violin plot, a red dot indicates the synchrony value of the LTM informed parcellation. Bottom and top edges of the narrow box in violin plot indicate 25 th and 75 th percentiles of the distribution, respectively. The central mark indicates the median. The whiskers extend to the most extreme data points that are not considered outliers $(1.5 \times$ interquartile range). Statistical analysis displayed by the violin plot demonstrates that BOLD signals in the LTM-informed parcellation are significantly more synchronous than expected due to chance $(P<0.05)$.

The results are shown in Fig. 8(d). Fig. 8(e) shows a mask that was generated within the same region from which random parcellations were derived to test whether these results were significantly different. The parcellation of occipital white matter was also validated using the second dataset of 13 subjects. In Fig. 8(f), the violin plot shows that the fMRI timecourses in the LTM-informed parcellation are significantly more synchronous than those in random parcellations.

\section{DISCUSSION}

In this work, we proposed a new method on the basis of GSP framework [20], we call filtered LTM reconstruction, to characterize the voxel-wise connectivity structure of human brains using fMRI data. In our approach, two key mathematical techniques are first formulated. First, an SVD-based filtering is used to suppress noise and global components, thereby enhancing the boundary features within connectivity profiles. As demonstrated by the connectivity matrices in our simulation studies (Fig. 3(b)\&3(c)), background connections between two voxel communities, that likely stem from common global components shared by the two communities and lead to blurring of their connectivity boundaries, are suppressed to relatively low levels by this SVD-informed filtering. Second, a novel LTM reconstruction is proposed to visualize connectivity structures that are implicitly encoded in a localized topoconnectivity graph. The resulting LTM images display spatial patterns of connectivity in which the image contrast of a voxel is dominated by its total connection weight with respect to neighboring voxels in cubic lattices of size $5 \times 5 \times 5$. The size of centrosymmetric cubic lattices for localizing the connectivity profile is determined as follows. First, for a smaller size $3 \times 3 \times 3$, the total connection weights have a limited range of values, which is unfavorable for identifying adjacent connectivity 
communities that have similar connection weights and thus have similar values in LTM images, while the difference of the connectivity communities can be more obvious for the size $5 \times 5 \times 5$. Second, a larger size (e.g., $7 \times 7 \times 7$ ) spoils the localization of connectivity profiles by too much, which confounds the capturing of local discontinuities, or the functional boundaries, in LTM reconstruction.

In vivo, the functional structures delineated by the boundaries in LTM images were compared with anatomical structures conveyed by $\mathrm{T}_{1} \mathrm{~W}$ images and DTI FA colored maps. Generally, the LTM images agree moderately with the two anatomic images. In particular, the LTM images reveal some subtle structures that are not observed in the anatomical images, which demonstrates that LTM images can offer distinctive information in functional structures that are not implied in anatomical structures. The convergency process of groupaverage LTM reconstruction are demonstrated in Section III in Supplementary material, which shows residual errors between the LTM images reconstructed with varying subsets of subjects and that reconstructed with all the 140 subjects. The LTM image reconstructed with 37 subjects can achieve a group consensus, which is informative for the group-average LTM reconstruction in the future. Note that the convergency speed of group-average LTM is dependent on the SNR of raw fMRI images that are related to parameters of MRI acquisitions.

Subtle connectivity structures delineated by group-average LTM images reconstructed from the fMRI dataset in HCP provide a basis for parcellation of a functional atlas of the human brain. The LTM contrast not only reveals functional structures, but also measures the synchrony of fMRI signals associated the voxels within a neighborhood. Voxels with low intensities in LTM images do not belong to any specific functional structures. As demonstrated in Fig. 4, a lot of voxels possess low intensities in the LTM images. However, the recently reported method based on functional connectivity gradients, which successfully parcellates subcortical structures, may not treat such voxels optimally [8]. For example, we considered parcellation of the hippocampus. As shown in Fig. 5 , numerous voxels around the core of the hippocampus have low intensities, which agrees with findings that the hippocampus has a centric stratified structure $[19,21]$. In this study, the voxels of low contrast in the LTM images are excluded to ensure that voxels contained in the parcellation atlas have synchronous BOLD signals.

Our first demonstration of LTM reconstruction is the parcellation of subcortical structures, which successfully recapitulates the well-known anatomic nuclei (Fig. 7(a)). Notably, the statistical analysis we performed to determine whether the synchrony of fMRI signals in LTM-informed parcellations are larger than expected due to chance is a further development of the existing statistical approach [8, 22]. Compared with the approach of random parcellation under relatively loose constraints $[8,22]$, our statistical analysis have two important improvements : (1), voxels constituting random parcellations are confined in a mask derived from the dilation of the LTM-informed parcellation with three layers of voxels and (2), most voxels in the random parcellations are required to be contained in the responding LTM-informed parcellation. Statistically, random parcellations under these two constraints would naturally have higher regional synchronies compared with the random parcellations without the two constraints. Hence, statistical analysis based on our constrained random parcellations should be more valid, suggesting that our statistical inference on synchrony of fMRI signals (Fig. 7(b)) is more valid.

The second demonstration of LTM reconstruction is the parcellation of occipital white matter, which provides insights to the emerging study of BOLD effects in white matter $[23,24]$. Clear functional boundaries in LTM images can be observed between gray matter and white matter, which suggests that BOLD signals of occipital white matter have independent origins. Furthermore, clear boundaries between gray matter and white matter can also be observed in the whole brain in LTM images. Based on this finding, we can exclude the possibility that BOLD signals in the white matter originate from effects of veins draining from the gray matter. The drainage effects from larger vessels in gray matter impair the spatial specificity of BOLD contrast in fMRI $[25,26]$, which have been proposed as potential sources of white matter fMRI signals [27].

LTM images of human brain demonstrate that functional structures also exist in various regions of white matter, which can provide two important principles for parcellation of white matter in a future study. First, voxels with low contrast intensities in LTM images should be removed from an atlas of white matter since BOLD signals associated with these voxels are not synchronous. Second, fine parcellations of white matter can be conducted using a combination of LTM images and diffusion tensor images. Improving the accuracy of atlas parcellation is essential to the region-level connectivity analysis of white matter since BOLD signals of white matter have low SNR and hence their detection is sensitive to small errors.

\section{CONCLUSION}

The proposed LTM reconstruction provides a novel method to derive voxel-wise functional structures within the human brain. Based on LTM images, accurate parcellations of functional structures in subcortical nuclei and occipital white matter were demonstrated in this study. The LTM reconstruction can be used to parcellate functional structures in other brain regions, which could offer a basis for more precise connectivity analyses at regional levels.

\section{REFERENCES}

1. [1] D. S. Bassett, and O. Sporns, "Network neuroscience," Nat Neurosci, vol. 20, no. 3, pp. 353-364, Feb 23, 2017.

2. [2] E. Bullmore, and O. Sporns, "Complex brain networks: graph theoretical analysis of structural and functional systems," Nat Rev Neurosci, vol. 10, no. 3, pp. 186-98, Mar, 2009.

3. [3] O. Sporns, "Contributions and challenges for network models in cognitive neuroscience," Nat Neurosci, vol. 17, no. 5, pp. 652-60, May, 2014.

4. [4] J. D. Medaglia, M. E. Lynall, and D. S. Bassett, "Cognitive network neuroscience," J Cogn Neurosci, vol. 27, no. 8, pp. 1471-91, Aug, 2015.

5. [5] T. A. Jarrell, Y. Wang, A. E. Bloniarz, C. A. Brittin, M. Xu, J. N. Thomson, D. G. Albertson, D. H. Hall, and S. W. Emmons, "The connectome of a decision-making neural network," Science, vol. 337, no. 6093, pp. 437-44, Jul 27, 2012. 
6. [6] C. T. Shih, O. Sporns, S. L. Yuan, T. S. Su, Y. J. Lin, C. C. Chuang, T. Y. Wang, C. C. Lo, R. J. Greenspan, and A. S. Chiang, "Connectomicsbased analysis of information flow in the Drosophila brain," Curr Biol, vol. 25, no. 10, pp. 1249-58, May 18, 2015.

7. [7] M. Bota, O. Sporns, and L. W. Swanson, "Architecture of the cerebral cortical association connectome underlying cognition," Proc Natl Acad Sci U S A, vol. 112, no. 16, pp. E2093-101, Apr 21, 2015.

8. [8] Y. Tian, D. S. Margulies, M. Breakspear, and A. Zalesky, "Topographic organization of the human subcortex unveiled with functional connectivity gradients," Nat Neurosci, vol. 23, no. 11, pp. 1421-1432, Nov, 2020

9. [9] K. V. Haak, A. F. Marquand, and C. F. Beckmann, "Connectopic mapping with resting-state fMRI," Neuroimage, vol. 170, pp. 83-94, Apr 15,2018

10. [10] D. S. Margulies, S. S. Ghosh, A. Goulas, M. Falkiewicz, J. M. Huntenburg, G. Langs, G. Bezgin, S. B. Eickhoff, F. X. Castellanos, M. Petrides, E. Jefferies, and J. Smallwood, "Situating the default-mode network along a principal gradient of macroscale cortical organization," Proc Natl Acad Sci U S A, vol. 113, no. 44, pp. 12574-12579, Nov 1, 2016

11. [11] X. Guell, J. D. Schmahmann, J. Gabrieli, and S. S. Ghosh, "Functional gradients of the cerebellum," Elife, vol. 7, Aug 14, 2018.

12. [12] D. Abramian, M. Larsson, A. Eklund, I. Aganj, C. F. Westin, and H. Behjat, "Diffusion-informed spatial smoothing of fMRI data in white matter using spectral graph filters," Neuroimage, vol. 237, pp. 118095, Aug 15, 2021.

13. [13] H. Behjat, N. Leonardi, L. Sornmo, and D. Van De Ville, "Anatomically-adapted graph wavelets for improved group-level fMRI activation mapping," Neuroimage, vol. 123, pp. 185-99, Dec, 2015.

14. [14] J. M. Huntenburg, P. L. Bazin, and D. S. Margulies, "Large-Scale Gradients in Human Cortical Organization,” Trends Cogn Sci, vol. 22, no. 1, pp. 21-31, Jan, 2018.

15. [15] L. Cerliani, R. M. Thomas, S. Jbabdi, J. C. Siero, L. Nanetti, A. Crippa, V. Gazzola, H. D'Arceuil, and C. Keysers, "Probabilistic tractography recovers a rostrocaudal trajectory of connectivity variability in the human insular cortex," Hum Brain Mapp, vol. 33, no. 9, pp. 200534, Sep, 2012.

16. [16] D. C. Van Essen, S. M. Smith, D. M. Barch, T. E. Behrens, E. Yacoub, K. Ugurbil, and W. U.-M. H. Consortium, "The WU-Minn Human Connectome Project: an overview," Neuroimage, vol. 80, pp. 6279 , Oct 15, 2013

17. [17] M. O. Irfanoglu, J. Sarlls, A. Nayak, and C. Pierpaoli, "Evaluating corrections for Eddy-currents and other EPI distortions in diffusion MRI: methodology and a dataset for benchmarking," Magn Reson Med, vol. 81, no. 4, pp. 2774-2787, Apr, 2019.

18. [18] H. Huang, C. Ceritoglu, X. Li, A. Qiu, M. I. Miller, P. C. van Zijl, and S. Mori, "Correction of B0 susceptibility induced distortion in diffusion-weighted images using large-deformation diffeomorphic metric mapping," Magn Reson Imaging, vol. 26, no. 9, pp. 1294-302, Nov, 2008.

19. [19] G. W. Kim, B. C. Kim, K. S. Park, and G. W. Jeong, "A pilot study of brain morphometry following donepezil treatment in mild cognitive impairment: volume changes of cortical/subcortical regions and hippocampal subfields," Sci Rep, vol. 10, no. 1, pp. 10912, Jul 2, 2020.

20. [20] D. I. Shuman, S. K. Narang, P. Frossard, A. Ortega, and P. Vandergheynst, "The emerging field of signal processing on graphs: Extending high-dimensional data analysis to networks and other irregular domains," IEEE Signal Processing Magazine, vol. 30, no. 3, pp. 83-98, 2013

21. [21] D. H. Adler, L. E. M. Wisse, R. Ittyerah, J. B. Pluta, S. L. Ding, L. Xie, J. Wang, S. Kadivar, J. L. Robinson, T. Schuck, J. Q. Trojanowski, M. Grossman, J. A. Detre, M. A. Elliott, J. B. Toledo, W. Liu, S. Pickup, M. I. Miller, S. R. Das, D. A. Wolk, and P. A. Yushkevich, "Characterizing the human hippocampus in aging and Alzheimer's disease using a computational atlas derived from ex vivo MRI and histology," Proc Natl Acad Sci U S A, vol. 115, no. 16, pp. 4252-4257, Apr 17, 2018.

22. [22] E. M. Gordon, T. O. Laumann, B. Adeyemo, J. F. Huckins, W. M. Kelley, and S. E. Petersen, "Generation and Evaluation of a Cortical Area Parcellation from Resting-State Correlations," Cereb Cortex, vol. 26, no. 1, pp. 288-303, Jan, 2016.

23. [23] J. C. Gore, M. Li, Y. Gao, T. L. Wu, K. G. Schilling, Y. Huang, A Mishra, A. T. Newton, B. P. Rogers, L. M. Chen, A. W. Anderson, and Z. Ding, "Functional MRI and resting state connectivity in white matter - a mini-review," Magn Reson Imaging, vol. 63, pp. 1-11, Nov, 2019.

24. [24] L. A. Grajauskas, T. Frizzell, X. Song, and R. C. N. D'Arcy, "White Matter fMRI Activation Cannot Be Treated as a Nuisance
Regressor: Overcoming a Historical Blind Spot," Front Neurosci, vol. 13, pp. 1024, 2019.

25. [25] A. T. Lee, G. H. Glover, and C. H. Meyer, "Discrimination of large venous vessels in time-course spiral blood-oxygen-level-dependent magnetic-resonance functional neuroimaging," Magn Reson Med, vol. 33, no. 6, pp. 745-54, Jun, 1995.

26. [26] R. Turner, "How much cortex can a vein drain? Downstream dilution of activation-related cerebral blood oxygenation changes," Neuroimage, vol. 16, no. 4, pp. 1062-7, Aug, 2002.

27. [27] M. Bianciardi, M. Fukunaga, P. van Gelderen, J. A. de Zwart, and J. H. Duyn, "Negative BOLD-fMRI signals in large cerebral veins," J Cereb Blood Flow Metab, vol. 31, no. 2, pp. 401-12, Feb, 2011. 